\title{
THE MAYER - VIETORIS SEQUENCE CALCULATING DE RHAM COHOMOLOGY
}

\author{
Arben Baushi \\ University " Ismail Qemali" of Vlora, Albania \\ arben.baushi@univlora.edu.al \\ baushiarben@gmail.com
}

\begin{abstract}
De Rham cohomology it is very obvious that it relies heavily on both topology as well as analysis. We can say it creates a natural bridge between the two. To understand and be able to explain what exactly de Rham cohomology is to the world of mathematics we need to know de Rham groups. This is the reasons to calculate the de Rham cohomology of a manifold. This is usually quite difficult to do directly. We work with manifold. Manifold is a generalization of curves and surfaces to arbitrary dimension. A topological space $\mathrm{M}$ is called a manifold of dimension $\mathrm{k}$ if :

- $M$ is a topological Hausdorff space

- M has a countable topological base.

- For all $\mathrm{m} \in \mathrm{M}$ there is an open neighborhood $\mathrm{U} \subset \mathrm{M}$ such that $\mathrm{U}$ is homeomorphic

to an open subset $\mathrm{V}$ of $\mathbb{R}^{\mathrm{k}}$.

There are many different kinds of manifolds like topological manifolds, $\mathbb{C}^{k}$ - manifolds, analytic manifolds, and complex manifolds, we concerned in smooth manifolds. A smooth manifold can described as a topological space that is locally like the Euclidian space of a dimension known. An important definion is homeomorphism. Let $\mathrm{X}, \mathrm{Y}$ be topological spaces, and let $\mathrm{f}: \mathrm{X} \rightarrow \mathrm{Y}$ e a bijection. If both $\mathrm{f}$ and the inverse function $\mathrm{f}^{-1}: \mathrm{X} \rightarrow \mathrm{Y}$ are continuous, then $\mathrm{f}$ is called a homeomorphism We introduce one of the useful tools for this calculating, the Mayer - Vietoris sequence. Another tool is the homotopy axiom. In this material I try to explain the Mayer - Vietoris sequence and give same examples. A short exact sequence of cochain complexes gives rise to a long exact sequence in cohomology, called the Mayer - Vietories sequence. Cohomology of the circle $\left(\mathrm{S}^{\wedge} 1\right)$, cohomology of the spheres $\left(\mathrm{S}^{\wedge} 2\right)$. Homeomorphism between vector spaces and an open cover of a manifold. we define de Rham cohomology and compute a few examples.
\end{abstract}

Indexing terms/Keywords : Mayer - Vietoris sequence; De Rham cohomology; manifold; differential forms; diffeomorfic ; exact ; close forms; cohomology class.

\section{INTRODUCTION}

Understanding the de Rham cohomology we may understand the types of spaces to work with. We work with manifold. Manifold is a generalization of curves and surfaces to arbitrary dimension. There are many different kinds of manifolds like topological manifolds, $\mathbb{C}^{k}$ - manifolds, analytic manifolds, and complex manifolds, we concerned in smooth manifolds. A smooth manifold can described as a topological space that is locally like the Euclidian space of a dimension known. The 'smooth' is relate to the differentiability of the maps that connect our space to the matching Euclidian space.

\subsection{Some preliminary concept}

Definition 1.1.[1] A topological space $M$ is called a manifold of dimension $k$ if:

- M is a topological Hausdorff space.

- $M$ has a countable topological base.

- For all $\mathrm{m} \in \mathrm{M}$ there is an open neighborhood $\mathrm{U} \subset \mathrm{M}$ such that $\mathrm{U}$ is homeomorphic

to an open subset $\mathrm{V}$ of $\mathbb{R}^{\mathrm{k}}$.

Definition 1. 2. Let $X, Y$ be topological spaces, and let $f: X \rightarrow Y$ be a bijection. If both $f$ and the inverse function $f^{-1}: Y \rightarrow X$ are continuous, then $f$ is called a homeomorphism. The homeomorphism $f$ maps each open set in $X$ to a unique open set in $\mathrm{Y}$. Whenever there exists a homeomorphism between two topological spaces, they are said to be homeomorphic . They have the same topological property (topologically the same).Under a homeomorphism, topological properties are preserved.

Example 1. (i) $(0 ; 1)$ and $(a, b)$ are homeomorphic, for example via the homeomorphism

$f(x)=a+(b-a) x$

(ii) The interval $[0 ; 1)$ and the circle $\mathbb{S}^{1}=\{z \in \mathbb{C}:|z|=1\}$ are not homeomorphic. There is a bijection $\mathrm{h}:[0 ; 1) \rightarrow \mathbb{S}^{1}$ given by $h(x)=e^{2 \pi i x}$, and $h$ is also continuous, but $h^{-1}$ is not continuous. That there is no homeomorphism between $[0 ; 1)$ and $\mathbb{S}^{1}$. 


\section{DEFINITION OF A SMOOTH MANIFOLD}

Definition 2.1 A smooth manifold is a pair $\left(M, A_{\max }\right)$ where $M$ is a manifold and $\mathbb{A}_{\max }$ maximal atlas.

Some time that called differentiable structure of $\mathrm{M}$.
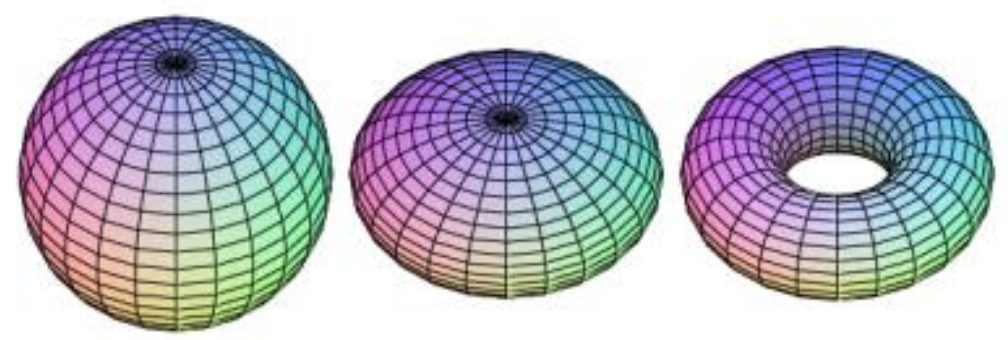

Fig. 1 . Some manifolds

Some examples of smooth manifold

(1) $\mathbb{R}^{n}$ is a smooth manifold .Atlas : $\phi: U=\mathbb{R}^{n} \rightarrow \mathbb{R}^{n}$ given by identity map.

(2) Any open subset $U$ of a smooth manifold $M$ is a smooth manifold. Given an atlas

$\mathbb{A}=\left\{\phi_{\alpha}: \mathrm{U}_{\alpha} \rightarrow \mathbb{R}^{\mathrm{n}}\right\}$ for $\mathrm{M}$, an atlas for $\mathrm{U}$ is $\left\{\phi_{\alpha}: \mathrm{U}_{\alpha} \cap \mathrm{U} \rightarrow \mathbb{R}^{\mathrm{n}}\right\}$

(3) $S^{n}=\left\{x_{1}^{2}+\cdots+x_{n+1}^{2}=1\right\} \subset \mathbb{R}^{n+1}$

( 4 ) (real projective space) $) \mathbb{R P}^{\mathrm{n}}=\left(\mathbb{R}^{\mathrm{n}+1}-\{(0, \ldots, 0)\}\right) / \sim$ where

$$
\left(\mathrm{x}_{0}, \mathrm{x}_{1}, \cdots, \mathrm{x}_{\mathrm{n}}\right) \sim\left(\mathrm{tx}_{0}, \mathrm{tx}_{1}, \ldots, \mathrm{tx}_{\mathrm{n}}\right), \mathrm{t} \in \mathbb{R}-\{0\}
$$

$\mathbb{R} \mathbb{P}^{n}$ is called the real projective space of dimension $n$. The equivalent class of $\left(x_{0}, x_{1}, \ldots, x_{n}\right)$ Is denoted by $\left[\mathrm{x}_{0}, \mathrm{x}_{1}, \ldots, \mathrm{x}_{\mathrm{n}}\right]$.

Let $\mathrm{U}_{0}=\left\{\mathrm{x}_{0} \neq 0\right\}$ with the coordinate chart $\phi_{0}: \mathrm{U}_{0} \rightarrow \mathbb{R}^{\mathrm{n}}$ is given by

$\left[\mathrm{x}_{0}, \mathrm{x}_{1}, \cdots, \mathrm{x}_{\mathrm{n}}\right]=\left[1, \frac{\mathrm{x}_{1}}{\mathrm{x}_{0}}, \cdots, \frac{\mathrm{x}_{\mathrm{n}}}{\mathrm{x}_{0}}\right] \mapsto\left(1, \frac{\mathrm{x}_{1}}{\mathrm{x}_{0}}, \cdots, \frac{\mathrm{x}_{\mathrm{n}}}{\mathrm{x}_{0}}\right)$

In the same way, take $U_{i}=\left\{x_{i} \neq 0\right\}$ and difine $\phi_{\mathrm{i}}: U_{\mathrm{i}} \rightarrow \mathbb{R}^{\mathrm{n}}$

\section{THE DE RHAM COMPLEX ON $\mathbb{R}^{\mathrm{n}}$}

In this section we difine de Rham cohomology and compute a few examples. This will turn out to be the most important diffeomorphism invariant of a manifold. We define $\Omega^{*}$ to be the algebra over $\mathbb{R}$ generated by $\mathrm{dx}_{1}, \ldots, \mathrm{dx}_{\mathrm{n}}$ with relation [3]

$$
\left\{\begin{array}{c}
\left(d x_{i}\right)^{2}=0 \\
d x_{i} d x_{j}=-d x_{j} d x_{i}, i \neq j
\end{array}\right.
$$

As a vector space over $\mathbb{R}, \Omega^{*}$ has basis

$1, d x_{i}, d x_{i} d x_{j}, d x_{i} d x_{j} d x_{k}, \ldots, d x_{1} \ldots d x_{n}$

$$
\mathrm{i}<\mathrm{j} \quad \mathrm{i}<\mathrm{j}<\mathrm{k}
$$

The $\mathbb{C}^{\infty}$ differential forms on $\mathbb{R}^{\mathrm{n}}$ are elements of $\Omega^{*}\left(\mathbb{R}^{\mathrm{n}}\right)=\left\{\mathbb{C}^{\infty}\right.$ function on $\left.\mathbb{R}^{\mathrm{n}}\right\} \otimes \Omega^{*}$

Defined as follows:

i) $\quad \mathrm{f} \in \boldsymbol{\Omega}^{0}\left(\mathbb{R}^{\mathrm{n}}\right)$, then $\mathrm{df}=\Sigma \frac{\partial \mathrm{f}}{\partial \mathrm{x}_{\mathrm{i}}} \mathrm{dx} \mathrm{x}_{\mathrm{i}}$

ii) $\quad \omega=\Sigma \mathrm{df}_{\mathrm{I}} \mathrm{dx}_{\mathrm{I}}$, then $\mathrm{d} \omega=\Sigma \mathrm{df}_{\mathrm{I}} \mathrm{dx}_{\mathrm{I}}$ 
EXAMPLE $1.1 \mathrm{If}=x d y$, then $d \omega=d x d y$

This $d$, called the exterior differentiation, is the ultimate abstract extension of the usual gradient, curl, and divergence of the vector calculus on $\mathbb{R}^{3}$, as below illustrates.

EXAMPLE 1.2 On $\mathbb{R}^{3}, \Omega^{0}\left(\mathbb{R}^{3}\right)$, and $\Omega^{3}\left(\mathbb{R}^{3}\right)$ are each 1-dimensional and $\Omega^{1}\left(\mathbb{R}^{3}\right)$ and $\Omega^{2}\left(\mathbb{R}^{3}\right)$ are each 3- dimensional over $\mathbb{C}^{\infty}$ function, so the following identification are possible:[2]

$\{$ function $\} \cong\{0-$ forms $\} \cong\{3-$ forms $\}$

$$
f \quad \leftrightarrow \quad f_{1} d x+f_{2} d y+f_{3} d z \quad \leftrightarrow f d x d y d z
$$

And

$\{$ vector fields $\} \cong\{1-$ forms $\} \cong\{2-$ forms $\}$

$\mathrm{X}=\left(f_{1}, f_{2}, f_{3}\right) \leftrightarrow d x+f_{2} d y+f_{3} d z \quad \leftrightarrow f_{1} d y d z-f_{2} d x d z+f_{3} d x d y$

On functions,

$$
d f=\frac{\partial f}{\partial x} d x+\frac{\partial f}{\partial y} d y+\frac{\partial f}{\partial z} d z
$$

On 1-forms,

$$
d\left(f_{1} d x+f_{2} d y+f_{3} d z\right)=
$$

$=\left(\frac{\partial f_{3}}{\partial y}-\frac{\partial f_{2}}{\partial z}\right) d y d z-\left(\frac{\partial f_{1}}{\partial z}-\frac{\partial f_{3}}{\partial x}\right) d x d z+\left(\frac{\partial f_{2}}{\partial x}-\frac{\partial f_{1}}{\partial y}\right) d x d z$

On 2-forms,

$d\left(f_{1} d y d z-f_{2} d x d z+f_{3} d x d y\right)=\left(\frac{\partial f_{1}}{\partial x}+\frac{\partial f_{2}}{\partial y}+\frac{\partial f_{3}}{\partial z}\right) d x d y d z$

So we have

$\mathrm{d}(0$-forms $)=$ gradient

$\mathrm{d}(1$-forms $)=$ curl,

$\mathrm{d}(2$-forms $)=$ divergence.

The wedge product of two differential forms, written $\tau \wedge \omega$ or $\tau \cdot \omega$ is defined as follows: if

$\tau=\sum f_{I} d x_{I}$ and $\omega=\sum g_{J} d x_{J}$ then

$$
\tau \wedge \omega=\sum f_{I} g_{J} d x_{I} d x_{J}
$$

Note that $\tau \wedge \omega=(-1)^{\operatorname{deg} \tau \operatorname{deg} \omega} \tau \wedge \omega$.

Proposition 3.1 $d$ is an antiderivation,

$$
d(\tau \cdot \omega)=(d \tau) \cdot \omega+(-1)^{\operatorname{deg} \tau} \tau \cdot d \omega
$$

Proof . By linearit it suffices to check on monomials

$\tau=\sum f_{I} d x_{I}, \omega=\sum g_{J} d x_{J}$

$d(\tau \cdot \omega)=d\left(f_{I} g_{J}\right) d x_{I} d x_{J}=d\left(f_{I}\right) g_{J} d x_{I} d x_{J}+f_{I} d g_{J} d x_{I} d x_{J}$

$=(d \tau) \cdot \omega+(-1)^{\operatorname{deg} \tau} \tau \cdot d \omega$

On the level of function

$d(f g)=(d f) g+f(d g)$ is simply the ordinary product rule.

Proposition 3.2. $d^{2}=0$.

Proof . [4]This is basically a consequence of the fact that the mixed partials are equal. On function, 
$d^{2} f=d\left(\sum_{i} \frac{\partial f}{\partial x_{i}} d x_{i}\right)=\sum_{i, j} \frac{\partial^{2} f}{\partial x_{j} \partial x_{i}} d x_{j} d x_{i}$

Here the factor $\frac{\partial^{2} f}{\partial x_{j} \partial x_{i}}$ are symmetric in $i, j$ while $d x_{i} d x_{j}$ are skew- symmetric in $\mathrm{i}, \mathrm{j}$; hence $d^{2} f=0$. On forms $\omega=f_{I} d x_{I}$, $d^{2} \omega=d^{2}\left(f_{I} d x_{I},\right)=d\left(d f_{I} d x_{I}\right)=0$

By the previous computation and the antiderivation property of $d$.

Now we give same examples exterior differentiation.

EXAMPLE 3.1 Consider function $f=x y$ and differential forms $\sigma=x d y+y d x$ and $\omega=z d x+x d y$.Calculate differential forms $d(f \omega)$, and $d(\sigma \wedge \omega)$

Solution. $d(f \omega)=d f \omega+f d \omega=d(x y)(z d x+x d y)+x y d(z d x+x d y)=$ $=(y d x+x d y) \wedge(z d x+x d y)+x y(d z \wedge d x+d x \wedge y)=x z d y \wedge d x+y x d x \wedge d y+x y d z \wedge d x+x y d x \wedge d y$

$=(2 x y-x z) d x \wedge d y+x y d z \wedge d x$.

$\sigma \wedge \omega=(x d y+y d x) \wedge(z d x+x d y)=x z d y \wedge d x+y x d x \wedge d y=(x y-x z) d x \wedge d y$

$d(\sigma \wedge \omega)=d((x y-x z) d x \wedge d y)=-x d z \wedge d x \wedge d y$

Note that $\sigma=d f$, hence $d(\sigma \wedge \omega)=d(d f \wedge \omega)=-d f \wedge d \omega$

The comlex $\Omega^{*}\left(\mathbb{R}^{n}\right)$ together with the differential operator $d$ is called de Rham complex on $\mathbb{R}^{n}$

Definition 3 . 1 [5] Let $\omega$ be a $k$ - form in an open subset $\subset \mathbb{R}^{n}$. If there is a (k-1)-form $\lambda$ in $U$ such that $\omega=d \lambda$, then $\omega$ is said to be exact in $U$.

If $\omega$ is of class a differentiable functions and $d \omega=0$, then is said to be closed.

However this Examles tell us that the closed forms wich are not exact.

EXAMPLE 3.2. Consider the 1-forms: $\eta=\frac{x d y-y d x}{x^{2}+y^{2}}$. Take $U=\mathbb{R}^{2}-\{0\}$

Proved that form is closed to $U=\mathbb{R}^{2}-\{0\}$ but it is not exat in

$$
U=\mathbb{R}^{2}-\{0\} .
$$

Solution. Fix $r>0$, take $\gamma(t)=(r \cos t, r \sin t) \quad(0 \leq t \leq 2 \pi)$

That $\gamma$ is a curve in $U=\mathbb{R}^{2}-\{0\}$. Take the value at point 0 and $2 \pi$ so $\gamma(0)=(r, 0)=\gamma(2 \pi)$

we get

$$
\partial \gamma=0
$$

Computing over $O$ and $\int \eta=2 \pi \neq 0$

We can see that $\eta$ is not exact form in $O=\mathbb{R}^{2}-\{0\}$ otherwise since $\partial \gamma=0$ that imply the integral ( 1$)$ it going to be 0 .

Definition. 3. 2 [3] The $k-t h$ de Rham cohomology of $\mathbb{R}^{n}$ is the vector space

$$
H_{D R}^{k}\left(\mathbb{R}^{n}\right)=\frac{\{\text { closed } k-\text { forms }\}}{\{\text { exact } k-\text { forms }\}}
$$

We sometime write that $H^{k}\left(\mathbb{R}^{n}\right)$

To distinguish between a form $\omega$ and its cohomology class,we denote the cohology class by [ $\omega$ ]

All definitions so far work equally well for any open subset $U$ of $\mathbb{R}^{n}$

\section{EXAMPLE 3.3}

(a) $\mathrm{n}=0$

$$
H^{k}\left(\mathbb{R}^{n}\right)= \begin{cases}\mathbb{R} & k=0 \\ 0 & k>0\end{cases}
$$

(b) $n=1$ 
Since (ker $d) \cap \Omega^{0}\left(\mathbb{R}^{1}\right)$ are the constant function

$$
H^{0}\left(\mathbb{R}^{1}\right)=\mathbb{R}
$$

On $\Omega^{0}\left(\mathbb{R}^{1}\right)$, kerd are all 1 - forms.

If $\omega=g(x) d x$ is a 1 - form,then by taking

$$
f=\int_{0}^{x} g(u) d u
$$

we find that

$$
d f=g(x) d x
$$

Therefore every 1 -form on $\mathbb{R}^{1}$ is exact so

$$
H^{1}\left(\mathbb{R}^{1}\right)=0
$$

\section{THE MAYER - VIETORIS SEQUENCE}

Let $M$ be a manifold and $U$ and $V$ both open subsets of $M$. A short exact sequences of a chain complexes give a rise a long exact sequence to the group cohomology correspond.

We take inclusions $i_{1}: U \rightarrow M$ and $i_{2}: V \rightarrow M$ gjithashtu $j_{1}: U \cap V \rightarrow U$ and $j_{2}: U \cap V \rightarrow V$

$h=\left(i_{1}{ }^{*}, i_{2}{ }^{*}\right)$ is a injective

$g=j_{1}{ }^{*}-j_{2}{ }^{*}$ is a syrjective

Now the sequences is short exact Mayer -Vietoris sequence

$$
0 \rightarrow \Omega^{*}(U \cup V) \stackrel{k}{\rightarrow} \Omega^{*}(U) \oplus \Omega^{*}(V) \stackrel{h}{\rightarrow} \Omega^{*}(U \cap V) \rightarrow 0
$$

From Homological Algebra we know that we get a long exact sequences of de Rham cohomology groups

$$
\rightarrow H^{n}(U \cup V) \stackrel{k}{\rightarrow} H^{n}(U) \oplus H^{n}(V) \stackrel{h}{\rightarrow} H(U \cap V) \stackrel{l}{\rightarrow} H^{n+1}(U \cup V) \rightarrow
$$

Where $l$ is the homomorphism, defined as. For $\omega \in \Omega^{n}(U \cup V)$ that have a property $d \omega=0$

Exist $\omega_{1} \in \Omega^{n}(U)$ and $\omega_{2} \in \Omega^{n}(V)$ that $\omega=j_{1}{ }^{*} \omega_{1}-j_{2}{ }^{*} \omega_{2}$ from that we get

$j_{1}{ }^{*} d \omega_{1}=j_{2}{ }^{*} d \omega_{2} \Rightarrow$ there is $\eta \in H^{n+1}(U \cup V)$ such that $i_{1}{ }^{*} \eta=d \omega_{1}$ and $i_{2}{ }^{*} \eta=d \omega_{2}$. So we get $l[\omega]=[\eta]$

We take a smooth manifold $M \times \mathbb{R}$, and let see a function $p_{1}: M \times \mathbb{R} \rightarrow M$ and

$p_{0}: M \rightarrow M \times \mathbb{R}$ such that $m \mapsto(m, 0)$

Lemma [6]. Given M And a maps defined above we have that $p_{1}{ }^{*}: H^{*}(M \times \mathbb{R}) \rightarrow \mathrm{H}^{*}(\mathrm{M})$ and $\mathrm{p}_{0}{ }^{*}: \mathrm{H}^{*}(\mathrm{M}) \rightarrow \mathrm{H}^{*}(\mathrm{M} \times \mathbb{R})$ are mutual invers, and in particular, $\mathrm{H}^{*}(\mathrm{M} \times \mathbb{R}) \cong \mathrm{H}^{*}(\mathrm{M})$

\section{Corrollary .}

$$
\mathrm{H}^{\mathrm{K}}(\text { point })=\mathrm{H}^{\mathrm{k}}\left(\mathbb{R}^{\mathrm{n}}\right)= \begin{cases}\mathbb{R} & \mathrm{k}=0 \\ 0 & \mathrm{k}>0\end{cases}
$$

Proof .We see that the statement is truue for $\mathrm{H}^{\mathrm{K}}$ (point). Than by inductions we get the corally

$$
\begin{gathered}
\mathrm{H}^{\mathrm{K}}(\text { point }) \cong \mathrm{H}^{\mathrm{k}}(\text { point } \times \mathbb{R})=\mathrm{H}^{\mathrm{K}}(\mathbb{R}) \\
\cong \mathrm{H}^{\mathrm{K}}(\mathbb{R} \times \mathbb{R})=\mathrm{H}^{\mathrm{K}}\left(\mathbb{R}^{2}\right) \\
\cong \\
\cong \mathrm{H}^{\mathrm{K}}\left(\mathbb{R}^{\mathrm{n}-1} \times \mathbb{R}\right)=\mathrm{H}^{\mathrm{K}}\left(\mathbb{R}^{\mathrm{n}}\right)
\end{gathered}
$$

\section{Conclusion}

Smooth manifolds can be characterized by an atlas, however these atlases can be very complex and hard to understand, so ideally we would like a simple characteristic of manifolds that we can easily work with. For the same cohomological 
space we can use a property to one for another. The linear approximation can be viewed as a generalizations of the linear approach to the graph of a function (tangent line of the graph of a function can be viewed as a linear approximation).

\section{REFERENCES}

I. Introduction to smooth Manifold : Jon M. Lee Publication Date: September 23, 2002 Edition: 1 p : 17-19

II. Introduction to differential forms : Artur Elezi Lecture note

III. Differential Forms in Algebraic Topology : Raoul Bott, Loring W. Tu 1982 by Springer-Verlag New York Inc $p: 13-16$

IV. Algebraic topology By Allen Hatcher Publication Date: December 3, 2001 Edition: 1 p: 249-251

V. Notesfor math 535a: differentialgeometry. www-bcf.usc.edu/ khonda/math535a/notes $p$ : 6-7.36-38

VI. Manifolds and Differential Geometry By Jeffrey Marc Lee p : 450

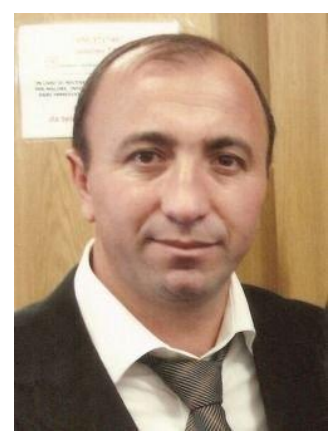

\section{Author' biography with Photo}

Lector , University “A. Moisiu” Durres . Albania 2007-2008

Lector , University “A. Xhuvani” Elbasan, Albani 2012-2016

Lector , University “I. Qemali” Vlora . Albania 2009 -2016

Maths Teacher Hight School Elbasan . Albania 1992-1994,2001-2007

1. Conference 5th ICHSS 2015 on Humanities and Social Sciences

ICHSS 2015 , 25-26 September, Rome. Italy : Micro Enterprises and New Trends Towards their Development , Arben Baushı, Vjollca Hasani, Alba Dumi

2. International Conference (ICRAE 2015) that will be held on University of Shkodra "Luigj Gurakuqi", $23^{\text {th }}-24^{\text {th }}$ October 2015. THE MAYER - VIETORIS SEQUENCE CALCULATING DE RHAM COHOMOLOGY, Arben Baushı,

3. VI Congress of the Mathematicians of Macedonia June 15-18, 2016

Characteristic of tiling generated by a root of polynomial

$$
P(x)=x^{4}-3 x^{3}+x^{2}-2 x-1
$$

4.The XI Annual International Meeting of "Alb-Shkenca" Institute 01-03 Shtator 2016 : Talk : Pisot and Salem numbers research to literatur

\section{Papers}

1.27, 28, 29 May 2016 Conference The 8-th INTERNATIONAL MULTIDISCIPLINARY CONFERENCE ON MEDICAL SPECIALTIES:

Fractal Application to Medicin

2. Introduction to topology, Book for Maths students (Albanian Langage) 2014 